\title{
Uso de Condom Feminino por Mulheres Infectadas pelo HIV
}

\author{
Female Condom Use among HIV-infected Women \\ Jarbas Magalhães*, Andréa da Silveira Rossi**, Eliana Amaral**
}

\begin{abstract}
RESUM0
Objetivo: avaliar a aceitabilidade, a adesão e a experiência com o uso de condom feminino (CF) entre mulheres infectadas pelo HIV.

Método: estudo descritivo prospectivo com 76 mulheres infectadas pelo HIV atendidas no CAISM/UNICAMP e no Centro Corsini de Campinas. Após entrevista de triagem e concordando em participar, as voluntárias receberam calendário para registro das relações sexuais e uso de condom masculino (CM). Após 30 dias, compareceram à visita de treinamento com colocação do CF em modelo pélvico, trazendo o diário do ciclo anterior, considerado controle. Aplicouse questionário estruturado após 30, 60 e 90 dias, recolhendo-se sempre o diário de registro das relações sexuais e uso de CF ou CM. Usaram-se os testes de $\chi^{2}$, exato de Fisher, McNemar e Friedman para amostras emparelhadas na análise estatística.

Resultados: predominaram as mulheres jovens, de baixa escolaridade, que moravam com o parceiro. Observou-se taxa de continuidade de uso de 52\%, ao longo de 90 dias. O uso de CF, em metade das relações sexuais em cada período de estudo, permaneceu estável nos 90 dias. Houve significativa diminuição da proporção média das relações sexuais desprotegidas (de 14\% para 6\%), sem uso de CM ou CF, aos 90 dias. As dificuldades iniciais no manuseio do $C F$ foram superadas com o tempo. Os casais sorodiscordantes tiveram maior proporção de relações protegidas que os casais soroconcordantes, porém a diferença não foi significativa. As mulheres que relataram uso prévio consistente de CM apresentaram número significantemente maior de relações protegidas com $\mathrm{CF}$.

Conclusões: a oferta do $C F$ foi capaz de reduzir as relações sexuais desprotegidas entre mulheres infectadas pelo HIV, que se mostraram motivadas e receptivas a este método.
\end{abstract}

PALAVRAS-CHAVE: Condom feminino. Anticoncepção. HIV. AIDS. Mulher. Prevenção.

\section{Introdução}

O condom feminino (CF) é dispositivo de poliuretano, na forma de bolsa transparente, macia e resistente, que mede $17 \mathrm{~cm}$ de comprimento por 7,8 cm de diâmetro, 0,42-0,53 $\mathrm{mm}$ de espessura. Contém dois anéis de borracha flexiveis, um menor que é móvel, do lado da parte fechada da bolsa, encaixando-se no colo do útero quando em

\footnotetext{
*Centro de Controle e Investigação Sorológica A.C. Corsini - Campinas/Brasil

**DTG - CAISM / Unicamp - Campinas/Brasil

Correspondência:

Jarbas Magalhães

Rua Alexandre Coelho 22 - J. Áurea

13.803-220 - Mogi Mirim - SP

Fone: (19) 3862-4545

email: jarbasmag@uol.com.br

Financiamento Coordenação Nacional de DST/AIDS, Ministério da Saúde
}

uso. O outro anel fica na outra extremidade, fixo, devendo permanecer exteriorizado durante o uso (na vulva), para impedir que toda a bolsa entre na vagina, fornecendo concomitantemente alguma proteção para a genitália externa. O condom é prélubrificado com fluido neutro, siliconado, não espermicida, conhecido como lubrificante "dimethicone", substância comumente usada na área médica, facilitando tanto a colocação do dispositivo quanto a penetração durante o ato sexual ${ }^{1}$.

Estudos in vitro demonstraram que o $\mathrm{CF}$ é barreira efetiva contra o HIV e outras DST, incluindo a hepatite $\mathrm{B}$ e o herpesvirus, podendo ser meio seguro para a prevenção destas infecções ${ }^{2}$. Pode ser usado também como método de barreira para contracepção e estima-se que a possibilidade de falha para gravidez possa chegar a menos de $5 \%{ }^{1}$. Não há evidências de trauma significante ou mudanças na flora vaginal associados ao seu uso ${ }^{3}$. 
O CF encontrará o seu melhor espaço como método complementar ao uso do condom masculino $(\mathrm{CM})$, quando o parceiro se recusar a utilizar o último, tiver dificuldades com o seu uso ou mesmo quando este não estiver disponivel ${ }^{4}$. O CF seria, então, método bastante interessante para casais em situações específicas. As mulheres infectadas pelo HIV, em princípio, estariam altamente estimuladas a utilizar um método de barreira, pela sua condição de serem potenciais transmissoras de vírus aos parceiros ou para evitar novas contaminações com HIV ou outras DST, além de prevenção da gravidez.

Sabe-se que as mulheres vivendo com HIV têm motivação especial para o uso de métodos de barreira, com aumento significativo do uso de CM após o diagnóstico de infecção. Apesar disso, um terço das sexualmente ativas não o utilizam, por razões de não-adesão ou falta de adaptação dos parceiros masculinos ${ }^{5}$. Paralelamente, sugere-se o uso de barreiras mesmo para casais concordantes, para evitar novos inóculos virais e/ou gravidez $z^{6}$. Nos casais discordantes, preveniria a transmissão sexual, agregando-se a possibilidade de evitar a gravidez, se esta for sua proposição reprodutiva.

A disponibilidade de outro método sob seu controle, na condição particular de mulheres infectadas pelo HIV, pode ser particularmente útil, reduzindo o número de relações sexuais sem proteção. Foi, então, proposto estudo prospectivo com o objetivo de avaliar a aceitabilidade, a adesão e a experiência com o uso do CF entre mulheres contaminadas pelo HIV.

\section{Pacientes e Métodos}

Foi realizado estudo clínico descritivo prospectivo, associado a estudo de conhecimento, atitude e prática ${ }^{7}$. Foram admitidas 63 mulheres infectadas pelo HIV, seguidas num serviço universitário (CAISM/UNICAMP), e 13 mulheres atendidas numa organização não governamental (Centro Corsini). O período de estudo se estendeu de maio de 2000 até julho de 2001 .

O cálculo amostral $(n=73)$ baseou-se em estudo prévio no mesmo serviço universitário sobre uso de contraceptivos por mulheres infectadas por $\mathrm{HIV}^{5}$, aceitando-se o valor de $\alpha=0,05$ e $\beta=0,05^{8}$. Foram incluídas mulheres infectadas pelo HIV com atividade sexual no último mês, que mostraram interesse em testar o CF e que concordaram em retornar segundo o agendamento proposto pelos pesquisadores (treinamento e visitas de seguimento de 30,60 e 90 dias). Foram excluídas aquelas que não conseguiram colocar o condom feminino no modelo pélvico durante a consulta de treinamento e aquelas cujos parceiros não concordaram previamente com sua participação na pesquisa.

Após serem instruídas sobre as várias etapas do estudo, as mulheres receberam e assinaram o consentimento livre e esclarecido. Depois, responderam a questionário de admissão com dados sociodemográficos, hábitos sexuais e antecedentes reprodutivos. Finalmente, receberam calendário para controle mensal do número de relações sexuais e uso de CM ou CF, sendo orientadas sobre o preenchimento deste.

Receberam nesta visita, $12 \mathrm{CM}$ para uso em 30 dias, número baseado na média de relações sexuais semanais destas mulheres, encontrada em estudo anterior ${ }^{5}$. Retornaram em um mês, trazendo o calendário preenchido, para a visita de treinamento. Os dados de freqüência de relações sexuais, uso de CM e eventualmente de CF do mês anterior ao treinamento foram considerados os dados basais, pré-intervenção.

Em seguida foi realizado treinamento prático de inserção do $\mathrm{CF}$, utilizando-se material didático com molde pélvico e $\mathrm{CF}$ e CM. A usuária foi então reorientada para preenchimento do calendário, incluindo o uso dos condons e eventuais relações desprotegidas. A partir desta visita, mensalmente foram fornecidas oito unidades de $\mathrm{CF}$ e doze de CM (ou quantidades maiores, a depender do calendário anterior), agendando-se o retorno para 30 dias. Nas consultas subseqüentes (30, 60 e 90 dias) foi aplicado questionário, para registro das impressões das mulheres sobre sua adaptação e aceitabilidade ao CF. Em todas as visitas foram recolhidos os calendários de relações sexuais preenchidos nos 30 dias anteriores. Foram excluídas da análise as 15 mulheres que abandonaram a pesquisa, no intervalo compreendido entre a triagem e o treinamento com o $\mathrm{CF}$.

Os questionários foram digitados em banco de dados criado no programa Epi-Info ${ }^{9}$. Tabelas de contingência foram elaboradas com o objetivo de descrever características demográficas da amostra. Para avaliar as possiveis mudanças na freqüência e padrão de relações sexuais praticadas pelos casais amostrados, foi utilizada a análise de variância $(\mathrm{ANOVA})^{10}$. As opiniões e percepções das mulheres foram avaliadas também através de tabelas de freqüências, as quais foram submetidas, quando necessário, a outros testes de hipóteses não paramétricos, como o teste para amostras emparelhadas de Wilcoxon e o teste de McNemar ${ }^{11}$. $\mathrm{Na}$ comparação entre o número de relações desprotegidas nas diferentes visitas foi também aplicada a análise de variância não paramétrica de Friedman. Quando o software Epi-Info ${ }^{9}$ tornou- 
se limitado para a análise estatística, o banco de dados foi transferido para o software SAS (Statistical Analysis System) onde recebeu novo tratamento. Estabeleceu-se o nível de significância de 0,05. Respeitaram-se os termos da Declaração de Helsinki ${ }^{12}$. O projeto foi aprovado pelo Comitê de Ética em Pesquisa da Faculdade de Ciências Médicas da UNICAMP e pelas respectivas comissões de ética das instituições envolvidas.

\section{Resultados}

Um total de 76 mulheres infectadas pelo HIV compareceu à consulta de triagem e 15 delas não voltaram para o treinamento de inserção do CF. O treinamento foi realizado por 61 mulheres, sendo que houve desistência de 13 mulheres entre 30 e 60 dias e de seis mulheres entre 60 e 90 dias. A taxa de continuidade ao final de 90 dias foi de $52 \%$.

As causas de desistências não foram conhecidas em quase metade das 15 mulheres que deixaram o estudo sem participar da visita de treinamento, apesar de tentativa de contatos telefônicos e por aerogramas. As causas mais freqüentes de abandono identificadas nesta fase foram relacionadas às dificuldades no trabalho e à grande distância dos locais de pesquisa. Dificuldades relacionadas ao parceiro não foram referidas neste período para justificar desistências, diferentemente do achado na fase pós-treinamento, quando a recusa do parceiro em usar CF ou não gostar do seu uso foi referida por um quarto das desistentes.

Metade das mulheres infectadas pelo HIV tinha menos de 30 anos e a grande maioria (82\%) havia cursado apenas o primeiro grau escolar. Pouco mais de dois terços delas eram casadas e apenas cerca de 3\% delas eram separadas ou divorciadas. A viuvez foi encontrada em pouco mais de $10 \%$ das mulheres infectadas pelo HIV. Quase $70 \%$ dessas mulheres viviam com o parceiro, $40 \%$ trabalhavam fora de casa e a metade de todas elas tinha baixa renda familiar.

Estudou-se a proporção de mulheres que praticaram relações desprotegidas na visita de treinamento e aos 30, 60 e 90 dias. Comparação múltipla entre as visitas mostrou que a diferença mais significativa no número de relações desprotegidas (em nivel de significância de 5\%) ocorreu entre a primeira e a terceira visita. Observamos também que a proporção de mulheres que praticavam relações desprotegidas diminuiu em cerca de três vezes na primeira e na segunda visita, quando comparada com a proporção da visita de treinamento. Permaneceu reduzida para a metade quando comparada com a terceira visita. O número de relações sexuais no mês variou consideravelmente, sendo que na segunda visita encontramos uma das mulheres infectadas pelo HIV que relatou ter praticado 28 relações desprotegidas (Tabela 1).

Tabela 1 - Proporção de mulheres que praticavam relações desprotegidas nas visitas de treinamento e aos 30,60 e 90 dias.

\begin{tabular}{lcccr}
\hline Visitas & Total de & \multicolumn{2}{c}{ Relações desprotegidas } \\
mulheres & $\begin{array}{c}\text { Relações } \\
\text { Min-máx }\end{array}$ & \multicolumn{2}{c}{ Mulheres } \\
\hline Treinamento & 59 & $1-25$ & 17 & \% \\
30 dias & 51 & $2-10$ & 5 & 10 \\
60 dias & 37 & $5-28$ & 3 & 8 \\
90 dias & 32 & $1-5$ & 5 & 16 \\
\hline
\end{tabular}

Teste não paramétrico de Friedman $p<0,01$

No ciclo controle que antecedeu o treinamento, a proporção média de relações sexuais com o CM foi bastante alta (84\%). Apenas $2 \%$ das mulheres usavam CF e 14\% das relações eram desprotegidas. Observou-se diminuição para a metade aos 30 e 90 dias e para um terço aos 60 dias, quando se compararam os períodos, em relação à proporção de relações desprotegidas do ciclo controle, antes do treinamento de inserção do $\mathrm{CF}$ (Figura 1).

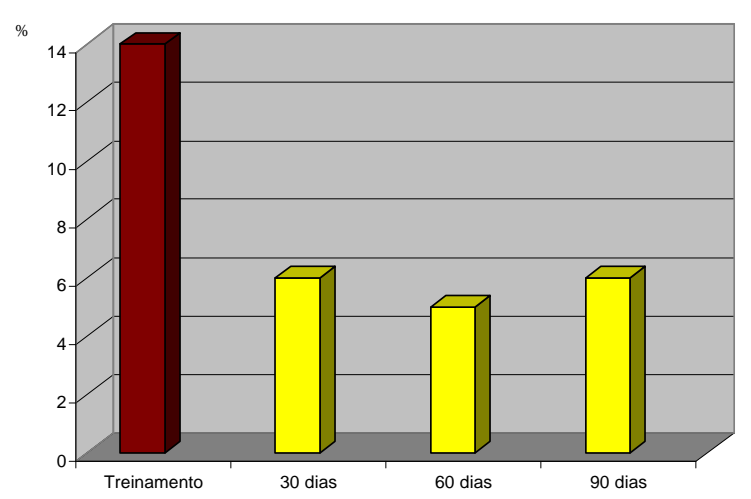

Figura 1 - Comparação da proporção média de relações sexuais desprotegidas na visita de treinamento com as dos períodos de 30,60 e 90 dias.

Houve significante migração do uso do CM para o uso do CF aos 30, 60 e 90 dias, quando se comparou o número total de relações sexuais praticadas em cada período do estudo (Figura 2). Na visita final do estudo, aos 90 dias, observou-se grande porcentagem $(81 \%)$ de relações protegidas entre as mulheres cujos parceiros eram soronegativos para o HIV (casais discordantes), porém a diferença não foi significativa quando se 
comparou com as que tinham parceiros soropositivos. As mulheres que relataram que seus parceiros usavam consistentemente o $\mathrm{CM}$ na fase anterior ao estudo foram as mulheres que apresentaram maior proporção (95\%) de relações protegidas na visita de 90 dias (Tabela 2).

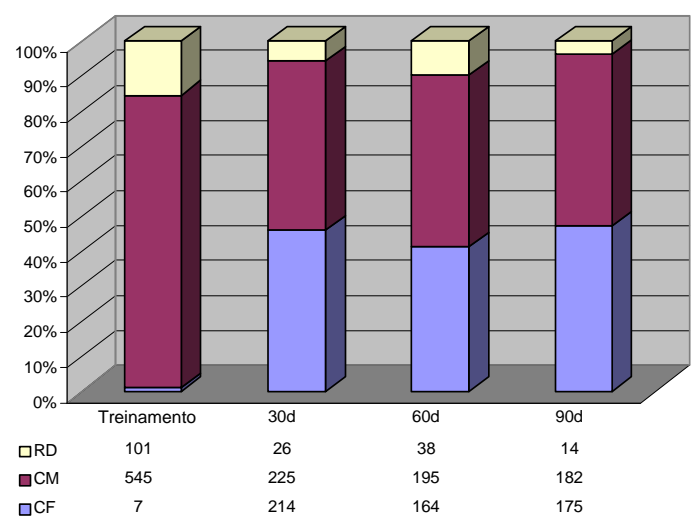

Figura 2 - Distribuição percentual dos tipos de relações sexuais (desprotegidas, condom masculino e feminino) nos diversos períodos. $\mathrm{RD}$, relações desprotegidas; $\mathrm{CM}$, condom masculino; CF, condom feminino.

Tabela 2 - Associação entre a condição sorológica do parceiro e uso consistente de condom no ciclo controle, com relações desprotegidas ao final do estudo $(n=32)$.

\begin{tabular}{|c|c|c|c|c|c|}
\hline & \multicolumn{4}{|c|}{$\begin{array}{c}\text { Relações } \\
\text { desprotegidas }\end{array}$} & \multirow{3}{*}{$p$} \\
\hline & \multicolumn{2}{|c|}{ Sim } & \multicolumn{2}{|c|}{ Não } & \\
\hline & $\mathbf{n}$ & $\%$ & $\mathrm{n}$ & $\%$ & \\
\hline \multicolumn{6}{|l|}{ Parceiro } \\
\hline Não tem parceiro fixo & 3 & 100,0 & 0 & 0,00 & 0,59 \\
\hline Parceiro infectado & 9 & 60,0 & 6 & 40,0 & \\
\hline Parceiro não infectado & 9 & 81,8 & 2 & 18,2 & \\
\hline Não sabe & 2 & 66,7 & 1 & 33,3 & \\
\hline \multicolumn{6}{|c|}{$\begin{array}{l}\text { Freqüência anterior de uso de } \\
\text { condom masculino }\end{array}$} \\
\hline Em todas as relações & 19 & 95,0 & 1 & 5,0 & $<0,01$ \\
\hline Outras freqüências & 4 & 33,3 & 8 & 66,7 & \\
\hline
\end{tabular}

As desvantagens mais relatadas pelas mulheres referentes ao $\mathrm{CF}$ foram dificuldade para colocar, achar feio e fazer barulho. Tais desvantagens foram significativamente superadas aos 90 dias de estudo. As vantagens mais comumente relacionadas pelas mulheres infectadas pelo HIV foram: a decisão de usar o CF pertencer à mulher e a dupla proteção contra gestação e DST/ HIV (Tabela 3).
Tabela 3 - Desvantagens e vantagens do condom feminino aos 30 e 90 dias por mulheres infectadas pelo HIV (\%).

\begin{tabular}{lccc}
\hline & 30 dias & 90 dias & $\mathbf{p}$ \\
\hline Desvantagens & & & \\
$\quad$ Dificuldade para colocar & 45 & 15 & 0,01 \\
Faz barulho & 28 & 07 & 0,03 \\
Feio & 45 & 34 & ns \\
Muito lubrificado & 17 & 14 & ns \\
Recusa do parceiro ao uso & 14 & 17 & ns \\
Dificuldade para retirar & 07 & 03 & ns \\
Vantagens & & & \\
A mulher decide quando usar & 31 & 38 & ns \\
Proteção gravidez e DST-HIV & 38 & 45 & ns \\
Uso somente na relação & 07 & 17 & ns \\
Fácil de usar & 21 & 28 & ns \\
\hline Teste de McNemar & ns: não significante (p>0,05) &
\end{tabular}

\section{Discussão}

Este é um dos primeiros estudos específicos sobre o uso de CF por mulheres infectadas pelo HIV. Seus resultados mostraram altas taxas de uso, continuidade e aceitabilidade em 90 dias de seguimento comparadas à visita de treinamento ou aceitação em iniciar o uso, com redução da proporção de relações desprotegidas.

Em estudo de fatores associados ao uso de CF em mulheres infectadas pelo HIV, 23\% das 90 mulheres incluídas recusaram-se a testar seu uso após visita de esclarecimento e de treinamento ${ }^{13}$. Foi observado pouco mais do que $31 \%$ de perda no presente estudo após aceitação inicial para testar o CF, sugerindo que novos esforços devam ser dedicados à tentativa de se obter melhor receptividade inicial, ou que este é o limite de aceitação deste dispositivo entre tais casais.

O uso de método combinado (outro contraceptivo associado a método de barreira) foi observado em 22 das 76 mulheres presentes na entrevista de triagem, sendo bem maior que o encontrado em estudo anterior do mesmo serviço, quando era utilizado por apenas $12 \%$ das mulheres infectadas pelo $\mathrm{HIV}^{5}$. Tais resultados, obtidos em período de três anos, parecem refletir a preocupação e a rotina destes serviços de ginecologia que atendem mulheres soropositivas em orientar a anticoncepção combinada em todas as consultas, sempre que possível.

A diminuição de um terço ou 50\% na proporção média de relações sexuais desprotegidas (sem uso de $\mathrm{CM}$ ou $\mathrm{CF}$ ) repete, neste grupo, achados de outros autores internacionais ${ }^{4,14}$ em mu- 
lheres não infectadas pelo HIV. Também se assemelha com a observação do estudo de aceitabilidade por usuárias dos serviços de saúde em diversas regiões do Brasil ${ }^{15}$.

Na Tailândia, uma população de mulheres trabalhadoras do sexo foi randomizada para oferecimento de CM apenas ou CF e CM. O uso de CM foi menor no grupo em que foram oferecidos os dois tipos de condons, quando comparado com o grupo que só recebeu o CM $(88,2$ e $97,5 \%)$. Houve também redução de $17 \%$ na proporção de relações desprotegidas, diferença que se manteve ao longo de um ano ${ }^{16}$. No presente estudo, encontrou-se redução ainda maior (35\%) nas relações sexuais com o uso de $\mathrm{CM}$ ao final dos 90 dias. O número médio de relações sexuais se manteve estável, mas houve substituição do uso de CM pelo CF nos períodos de 30 a 90 dias.

Em estudo envolvendo 919 mulheres de clínica de doenças sexualmente transmissiveis, com seguimento de seis meses incluindo diário de relações sexuais, 731 mulheres relataram ter usado o CF pelo menos uma vez durante o primeiro mês de seguimento, o que corresponde a uma taxa de uso de $79 \%$. No final do estudo, $8 \%$ das mulheres usaram exclusivamente o CF e 15\% usaram exclusivamente o CM. O uso dos dois tipos de condons foi relatado por $73 \%$ das mulheres e apenas 3\% não usaram método de barreira ${ }^{4}$. Embora os resultados deste estudo sejam semelhantes aos nossos, não encontramos uso exclusivo de CF por nenhuma das 32 mulheres infectadas pelo HIV que concluíram a observação, aos 90 dias. Isso ocorreu para alguns casos em um ou outro ciclo, de maneira esporádica.

Neste estudo com mulheres infectadas pelo HIV encontramos taxas altas de aceitabilidade do $\mathrm{CF}$, possivelmente por serem mulheres bastante estimuladas ao uso de métodos de barreira. Em estudo realizado com 90 mulheres infectadas pelo HIV, em Wisconsin, EUA, encontrou-se taxa de 77\% de uso, sendo que apenas 30\% das mulheres estavam usando o condom ao final do estudo. Os autores observaram maior taxa de uso do CF entre mulheres que tinham múltiplos parceiros e que relataram poucas relações desprotegidas no período anterior à sua oferta ${ }^{13}$.

Em estudos relacionando o tipo de parceiro sexual e a consistência de uso de CF, observou-se maior uso entre mulheres com parceiros fixos do que naquelas com um novo parceiro ou com um parceiro ocasional ${ }^{4}$. Na nossa amostra, os casais sorodiscordantes (parceiro masculino soronegativo) apresentaram uma maior proporção de relações protegidas, porém não houve diferença significativa quando comparados com os casais soroconcordantes.
No presente estudo, entre as 32 mulheres que compareceram aos 90 dias, observou-se significativa predominância de uso entre aquelas cujos parceiros relataram uso prévio consistente de CM. Tais resultados sugerem que a experiência de proteção na relação sexual, anterior à intervenção de oferta de $\mathrm{CF}$, seja fator importante na continuidade de uso, pois foram obtidos ao final dos 90 dias. Este mesmo fato foi observado entre as mulheres brasileiras usuárias de serviços de saúde que aceitaram utilizar o $\mathrm{CF}^{15}$.

A continuidade de uso do CF baseou-se na proporção entre as mulheres presentes na visita de 90 dias $(n=32)$ e aquelas presentes na visita de treinamento $(n=61)$. A aplicação dessa forma de cálculo levou a taxas de continuidade de 52\%. Esta taxa de continuidade é maior que os 30\% observados em outro estudo sobre infectadas pelo HIV ${ }^{17}$. Diversos autores apontam para a importância da forma e da qualidade da oferta dos serviços de saúde na obtenção de altos índices de aceitabilidade dos métodos de barreira, entre eles o $\mathrm{CF}^{17}$. De fato, forte determinante de adesão a tratamento ou métodos é a boa relação do usuário com o serviço. Enfoque de atenção integral à saúde, com o apoio de equipe multi e interdisciplinar, é a rotina dos serviços onde o estudo foi realizado, o que é particularmente necessário para estas mulheres. Isto, certamente, tem garantido maior adesão das usuárias ao serviço e às intervenções.

As dificuldades no uso do CF foram bastante prevalentes por ocasião da visita de 30 dias, quando praticamente a metade das mulheres relatou dificuldade para colocação e cerca de um terço delas relatou desconforto e barulho durante a relação sexual. Notamos que foi significante o progresso das mulheres no manuseio do $\mathrm{CF}$, com diminuição nas referências de dificuldade para colocar, desconforto e barulho no momento do ato sexual. Resultado semelhante ocorreu no trabalho conduzido na Costa Rica, numa população de trabalhadoras do sexo, na qual os problemas mais comuns nas primeiras duas semanas foram: dificuldade para inserir (61\%) e desconforto durante a relação sexual (43\%). Entretanto, após quatro semanas, houve redução destes problemas para 22 e $25 \%$, respectivamente ${ }^{17}$.

Se consideramos necessário um tempo de adaptação ao método, que propicie familiaridade e facilitação do uso, é essencial incluir retornos de avaliação e/ou orientação precoces quando o casal inicia o uso deste método. Para isso, os serviços que os oferecem precisam ter equipe acolhedora, capacitada especificamente nas questões práticas que acompanham o uso do método e que permita acesso rápido, se houver dificuldades com o uso. 
Entre as limitações deste estudo, a mais importante pareceu ser o número de perdas de seguimento de mulheres no início da pesquisa. Procuramos minimizar as perdas estudando atentamente os motivos de abandono, que se concentraram em motivos pessoais, como dificuldades para deixar o trabalho e comparecer às visitas propostas. Em contrapartida, é uma das primeiras observações prospectivas mundiais sobre o $\mathrm{CF}$ feitas exclusivamente numa população infectada pelo HIV. Até onde pudemos buscar, é o primeiro estudo brasileiro nesta população de mulheres.

Nossos achados coincidem com aqueles de estudos prévios, em que se observou que quando lhes é dada possibilidade de escolher entre os métodos de barreira, a maioria das mulheres tentará o uso do $\mathrm{CF}$, diminuindo significativamente a proporção de relações sexuais desprotegidas. Embora nossos resultados sejam de uma população específica de mulheres infectadas pelo HIV e não possa ser generalizado, parece possivel inferir também que, conforme os casais ganham confiança e experiência, atitudes positivas em relação ao método se tornam mais prevalentes, aumentando as taxas de continuidade de uso e aceitabilidade.

Em particular, quase 70\% deste grupo de 32 mulheres que mantiveram o uso até 90 dias, referiu querer continuar usando o CF. Esta proporção é a mesma observada entre as mulheres brasileiras dos serviços de saúde das diversas regiões ${ }^{15}$. Sendo este um insumo caro, de difícil acesso pelas mulheres brasileiras da população geral, continuará sendo necessário definir os grupos que mais possam se beneficiar de sua distribuição. Este estudo fornece subsídios para reforçar a inclusão das mulheres infetadas pelo HIV e, por extensão, as outras mulheres mais expostas a este risco, entre aquelas que devem ser agraciadas com sua distribuição. A re-utilização do $\mathrm{CF}$ após lavagem e lubrificação é outra possibilidade que está sendo considerada cuidadosamente, com resultados promissores ${ }^{18}$. Entretanto, as avaliações realizadas foram controladas em laboratório e podem não representar o risco real de deterioração do dispositivo na sua utilização pelas mulheres em suas residências.

Esforços adicionais são necessários para maximizar o acesso a todos os métodos anticoncepcionais. Neste contexto, o condom feminino deve receber alta prioridade, por ser método controlado pela mulher e que proporciona proteção contra gravidez e DST/HIV. Entre estes esforços, incluem-se a redução de custo, a avaliação adequada da reutilização e a definição das características dos grupos populacionais que mais se beneficiarão como sua disponibilidade.

\section{ABSTRACT}

Purpose: to evaluate acceptability, adhesion and experience with the use of female condom (FC) among HIV-infected women.

Methods: prospective descriptive study with 76 HIV-infected women under care at CAISM/UNICAMP and Centro Corsini, both in Campinas. After a screening interview and agreeing to participate, the volunteers received a diary to register their intercourses and correspondent use of male condom $(M C)$. After 30 days, they returned to a training visit when the FC was inserted in a pelvic model, also bringing their diary related to the previous cycle, considered a control cycle. A structured questionnaire was used at 30, 60 and 90 days, also with the respective diary on sexual intercourse and use of MC and FC kept for posterior data entry. $\chi^{2}$, Fisher's exact, McNemar and Friedman tests were applied statistical analysis of paired samples.

Results: there was a predominance of young women, with low schooling, living with their partner. Rate of continuity was $52 \%$ after 90 days. The use of FC in half the intercourses for each time period remained stable over the 90-day interval. There was a significant reduction in unprotected intercourses (from 14 to 6\%), without use of FC or MC, at the end of the period. The initial difficulties in handling the device were overcome. Serodiscordant couples had more protected intercourses than concordant couples, but the difference did not reach statistical significance. Women reporting consistent previous use of MC had more protected sex using $F C$.

Conclusions: the offer of the female condom was able to reduce unprotected intercourses in HIV-infected women, which were highly motivated and receptive for the new method.

KEYWORDS: Female condom. Contraception. HIV. AIDS. Women. Prevention.

\section{Agradecimentos}

Regina Maria Barbosa, NEPO/UNICAMP, pelo estímulo e auxílio na discussão do projeto e avaliação crítica na análise e apresentação dos dados.

Edson Giacomini, pelo auxílio na análise dos dados.

\section{Conflito de interesses:}

Não mencionado 


\section{Referências}

1. World Health Organization. The female condom: a review. Geneva, 1997. (World Bank Special Programme of Research, Development and Research Training in Human Reproduction).

2. Drew WL, Blair M, Miner RC, Conant M. Evaluation of the virus permeability of a new condom for a woman. Sex Transm Dis 1990; 17:110-2.

3. Soper DE, Brockwell NJ, Dalton HP. Evaluation of the effects of a female condom on the female lower genital tract. Contraception 1991; 44:21-9.

4. Macaluso M, Demand MJ, Artz LM, Hook EW $3^{\text {rd }}$. Partner type and condom use. Aids 2000; 14:537-46.

5. Magalhães J, Amaral E, Giraldo PC, Simões JA. HIV infected women: impact on contraception. Contraception 2002; 66:87-91.

6. Blocker ME, Cohen MS. Biologic approaches to the prevention of sexual transmission of human immunodeficiency virus. Infect Dis Clin North Am 2000; 14:983-99.

7. Warwick DP, Lininger AC. Introduction. In: Warwick DP, editor. The Sample Survey: theory and practice. New York: McGraw Hill; 1975. p.4-19.

8. Lachenbruch PA. On the sample size for studies based upon McNemar's test. Stat Med 1992; 11:1521-5.

9. Dean AG, Dean JA, Burton, AH, Dicker, RC. EpiInfo [computer program]. Version 5.0: a word processing, database, and statistics program for epidemiology on microcomputers. Atlanta: USD; 1990.
10. Scheffe $H$. The analysis of variance. $1^{\text {st }}$ ed. New York: John Wiley; 1959; p.477.

11. Armitage P. Statistical methods in medical research. $1^{\text {st }}$ ed. New York: John Wiley and Sons; 1974.

12. Organización Panamericana de la Salud. Asamblea Médica Mundial. Declaración de Helsinki. Bol Oficina Sanit Panam 1990; 108:626-9.

13. Kalichman SC, Rompa D, Cage M. Factors associated with female condom use among HIVseroposite women. Int J STD AIDS 2000; 11:798803.

14. Musaba E, Morrison CS, Sunkutu MR, Wong EL. Long-term use of the female condom among couples at high risk of human immunodeficiency virus infection in Zambia. Sex Transm Dis 1998 25:260-4.

15. Ministério da Saúde. Secretaria de Políticas de Saúde. Coordenaçäo Nacional de DST e Aids. Aceitabilidade de condom feminino em contextos sociais diversos: relatório final de pesquisa. Brasília: Ministério da Saúde; 1999. p.55.

16. Fontanet AL, Saba J, Chandelying V, et al. Protection against sexually transmitted diseases by granting sex workers in Thailand the choice of using the male or female condom: results from a randomized controlled trial. AIDS 1998; 12:1851-9.

17. Madrigal J, Schifter J, Feldblum J. Female condom acceptability among sex workers in Costa Rica. AIDS Educ Prev 1998; 10:105-13.

18. Potter B, Gerofi J, Pope M, Farley T. Structural integrity of the polyurethane female condom after multiple cycles of disinfection, washing, drying, and relubrication. Contraception 2003; 67:65-72.

Recebido em: 23/4/2003 Aceito com modificações em: 25/6/2003 